\title{
Gradiente altitudinal y su influencia en las características edafoclimáticas de los bosques tropicales
}

\section{Altitudinal gradient and its influence on the edofoclimatic characteristics of tropical forests}

\author{
Hipolito Murga-Orrillo", Manuel Fernando Coronado Jorge ${ }^{2}$, Carlos Abanto-Rodríguez ${ }^{3}$ y Francisco De Almeida Lobo ${ }^{4}$ \\ 1 Universidad Nacional Autónoma de Alto Amazonas. \\ Yurimaguas, Alto Amazonas, Loreto, Perú. \\ 2 Universidad Nacional de San Martín. Tarapoto, Perú. \\ 3 Instituto de Investigaciones de la Amazonía * * Autor de correspondencia. hmurga@unaaa.edu.pe \\ Peruana. Ucayali, Perú. \\ 4 Universidade Federal de Mato Grosso. Cuiabá, \\ MT, Brasil.
}

\section{RESUMEN}

Los gradientes altitudinales pueden generar límites ambientales, influyendo en el crecimiento y desarrollo de las especies vegetales. Se tuvo por objetivo identificar las variaciones edafoclimáticas en gradientes altitudinales y su influencia en los bosques tropicales. Se buscaron artículos de investigación con ámbito geográfico entre $27^{\circ} \mathrm{N}$ y $27^{\circ} \mathrm{S}$ de latitud, con influencia de gradientes altitudinales entre 0 m y 5000 m. Se sistematizó la información, organizándose en temáticas del clima, en las propiedades fisicoquímicas y biológicas del suelo, y en el comportamiento de las especies forestales. Se determinó que la temperatura media anual (TMA) disminuye conforme aumenta la altitud; y la precipitación anual (PA) presenta máximos pluviales en altitudes medias. Los valores del pH del suelo disminuyen hasta $3000 \mathrm{~m}$, aumentando sobre esta altitud; sin embargo, la materia orgánica, carbono orgánico, carbono total (CT), nitrógeno total (NT) y la relación C/N del suelo aumentan hasta $3000 \mathrm{~m}$; sobre esta altitud los valores disminuyen. El potasio (K), el calcio (Ca), el magnesio $(\mathrm{Mg})$, el fierro (Fe), y el aluminio (Al) del suelo disminuyen con el aumento de la altitud por influencia de las bajas temperaturas y humedad del suelo (HS). La variación edafoclimática en los gradientes altitudinales interactúa con las especies forestales, afectando las respuestas fisiológicas, disminuyendo su crecimiento y altura con el aumento de la altitud; del mismo modo disminuye su densidad y diversidad con el aumento de la altitud. Las especies forestales de los trópicos prosperan en amplios o estrechos límites altitudinales y edafoclimáticos, por lo que identificar estos límites es fundamental no solo en términos ecológicos, sino también políticos y económicos, para diseñar políticas efectivas de uso de la tierra y de conservación.

PALABRAS CLAVE: altitud, carbono, clima, precipitación, suelo, temperatura.

\section{ABSTRACT}

Altitudinal gradients can generate environmental limits, influencing the growth and development of plant species. The aim was to understand the edaphoclimatic variation in altitudinal gradients, and its influence on tropical forests. Research articles with a geographic scope between $27^{\circ} \mathrm{N}$ and $27^{\circ} \mathrm{S}$ latitude were searched, with influence of altitudinal gradients between $0 \mathrm{~m}$ and $5000 \mathrm{~m}$; The information was systematized, organizing it in climatic issues, in physicochemical and biological properties of the soil, and in the behavior of forest species. It was determined that the mean annual temperature (TMA) decreases as the altitude increases, and the annual precipitation (PA) presents maximum pluvial at mid altitudes; soil $\mathrm{pH}$ values decrease up to $3000 \mathrm{~m}$, increasing above this altitude; however, organic matter, organic carbon, total carbon (TC), total nitrogen (NT) and the $\mathrm{C} / \mathrm{N}$ ratio of the soil increase up to $3000 \mathrm{~m}$ a.s.l., above this altitude the values decrease; potassium $(\mathrm{K})$, calcium $(\mathrm{Ca})$, magnesium $(\mathrm{Mg})$, iron $(\mathrm{Fe})$, and aluminum $(\mathrm{Al})$ in the soil decrease with increasing altitude due to the influence of low temperatures and soil moisture (HS). The edaphoclimatic variation in the altitudinal gradients interacts with the forest species, affecting the physiological responses, decreasing their growth and height with increasing altitude; in the same way its density and diversity decreases with increasing altitude. The forest species of the tropics thrive in wide or narrow elevational and edaphoclimatic limits, so identifying these limits is essential not only in ecological terms, but also in political and economic terms, to design effective land use and conservation policies.

KEYWORDS: altitude, carbon, climate, rainfall, soil, temperature. 


\section{INTRODUCCIÓN}

Los bosques tropicales se encuentran distribuidos en gradientes altitudinales, los cuales presentan complejas topografías (Wilson y Rhemtulla, 2018), van desde el nivel del mar hasta más de 3500 m (Asner y Martin 2016). Considerando la proximidad a la línea ecuatorial, se encuentran entre los trópicos de Cáncer y Capricornio entre latitudes de $0^{\circ}$ a $23.45^{\circ} \mathrm{N} / \mathrm{S}$, respectivamente. De modo similar, Holdridge (1967) clasifica el clima de los bosques tropicales, según la latitud, en tropical, entre $0^{\circ}$ y $13^{\circ} \mathrm{N} / \mathrm{S}$; y subtropical, entre $13^{\circ}$ y $27.5^{\circ} \mathrm{N} / \mathrm{S}$. En relación con la altitud, los clasifica en premontanos, entre 1000 m y 2000 m; bajo montanos entre $2000 \mathrm{~m}$ y $3000 \mathrm{~m}$; y montanos entre $3000 \mathrm{~m}$ y $4000 \mathrm{~m}$. Estos bosques presentan variaciones anuales de temperatura media y precipitación: en premontano, de $18{ }^{\circ} \mathrm{C}$ a $24{ }^{\circ} \mathrm{C}$ y de $1200 \mathrm{~mm}$ a 8000 $\mathrm{mm}$; en bajo montano, de $12{ }^{\circ} \mathrm{C}$ a $18{ }^{\circ} \mathrm{C}$ y de $8000 \mathrm{~mm}$ a $6000 \mathrm{~mm}$; y en montano de $6{ }^{\circ} \mathrm{C}$ a $12{ }^{\circ} \mathrm{C}$ y de $6000 \mathrm{~mm}$ a $4000 \mathrm{~mm}$, respectivamente (Aybar-Camacho et al., 2017). Sin embargo, en los valles interandinos, las áreas están protegidas por montañas, que hacen variar la temperatura media anual (TMA) y la precipitación anual (PA), presentando valores menores (Villar et al., 2010).

Los gradientes altitudinales influyen en las condiciones climáticas, en las propiedades y en la biología del suelo, en la estructura poblacional y en la fisiología de las especies. Además, actúan como filtros, causando heterogeneidad ambiental, interactuando con los procesos ecológicos, biogeográficos y evolutivos en escala local y temporal (Terra et al., 2015; Asner et al., 2016; Asner et al., 2017). El suministro de nutrientes, el gradiente altitudinal y el clima regulan los procesos fisiológicos de las especies de los bosques tropicales (Bahar et al., 2017). Los suelos de los bosques tropicales presentan grandes entradas de nutrientes atmosféricos y poco se sabe sobre el aumento de estos nutrientes en gradiente altitudinal (Baldos, Corre y Veldkamp, 2015). Falta comprender la variación del almacenamiento del carbono orgánico en la biomasa del bosque tropical, en la materia orgánica y CT del suelo (De la Cruz-Amo et al., 2020); igualmente, poco se sabe sobre la distribución de especies forestales a lo largo del gradiente altitudinal y las condiciones climáticas.

La presión antrópica sobre los bosques tropicales es cada vez más intensa, su protección en gradiente altitudinal es esencial para conservar la diversidad de especies forestales, siendo en la actualidad, prioridad de conservación global (Wilson y Rhemtulla, 2018).

En este documento se analizan las relaciones de gradiente altitudinal en latitudes tropicales con la TMA, la $\mathrm{PA}$, el pH, la fertilidad, el carbono, la relación $\mathrm{C} / \mathrm{N}$, los hongos, las baterías y las archaeas del suelo, la estructura poblacional de las plantas y de sus respuestas fisiológicas. Se plantea la hipótesis de que los gradientes altitudinales tienen influencia en los componentes edáfoclimáticos, en la vegetación y en la biología del suelo.

\section{OBJETIVOS}

Comprender la variación climática y edáfica en gradientes altitudinales y su influencia en los bosques tropicales. Generar interpretaciones de informaciones técnicas a fin de ser consideradas en la gestión de los recursos naturales.

\section{MATERIALES Y MÉTODOS}

Entre marzo y julio de 2020, se realizó una búsqueda y selección detallada de artículos de investigación, desarrollados en bosques tropicales entre $27^{\circ} \mathrm{N}$ y $27^{\circ} \mathrm{S}$ de latitud, con influencia de gradientes entre $0 \mathrm{~m}$ y $5000 \mathrm{~m}$ de altitud. Los artículos fueron buscados en Google académico y en las bases de datos de Scopus, Web of Science, Science Direct y Springer, utilizando palabras clave como "elevation gradient, andean tropical, tropical montane, altitudinal gradients, tropical forests, biogeography tropical, forests soils, tropical mountain, forest communities, rainforest, forest structure y temperature forest". La información obtenida fue filtrada a un total de 52 artículos, a los cuales se realizó un análisis sistematizado de sus resultados y, según la influencia del gradiente altitudinal en las variables edafoclimáticas y en las características de los bosques, se organizó la información en: a) variaciones de la temperatura y precipitación pluvial, b) propiedades fisicoquímicas y biológicas del suelo, c) estructura poblacional del bosque tropical y sus respuestas fisiológicas. 
Se analizó e interpretó la información, lo que permitió elaborar una propuesta del comportamiento en el perfil altitudinal de la TMA y la PA, la HS, el pH, el CT, el fosforo total (PT), el NT, el Ca, el K, el Fe, el Al, el Mg, la relación $\mathrm{C} / \mathrm{N}$, los hongos, las bacterias y las archaeas en el suelo; además, de la altura y el crecimiento de árboles en bosques tropicales. Con los datos de la tabla 1 se elaboraron gráficos de dispersión y tendencia utilizando el software estadístico Rstudio (R Core Team, 2021).

\section{RESULTADOS Y DISCUSIÓN}

\section{Influencia de la temperatura y precipitación pluvial en la vegetación}

Las PA varían con la altitud, alcanzando máximos pluviales de $5302 \mathrm{~mm}$ a los $1713 \mathrm{~m}$ (Oliveras et al., 2020) y de 5300 mm a los 1527 m (Bahar et al., 2017); el mínimo pluvial registrado fue de $760 \mathrm{~mm}$ entre altitudes de $4100 \mathrm{~m}$ y 4400 $\mathrm{m}$ (Llambí et al., 2020). En la fig. 1b se observa el comportamiento de la PA de $0 \mathrm{~m}$ a más de $4000 \mathrm{~m}$ de altitud, mostrando una tendencia decreciente a partir de las máximas PA; sin embargo, con respecto a la TMA presentan disminución térmica lineal por el aumento de la altitud en el gradiente altitudinal (Fig. 1a). La disminución térmica por cada $100 \mathrm{~m}$ de incremento de la altitud, entre $2865 \mathrm{~m}$ y 3550 , $\mathrm{m}$ fue de $-0.48^{\circ} \mathrm{C}$ (Wang Yang, Deslauriers y Bräuning, 2014); entre $1952 \mathrm{~m}$ y $3550 \mathrm{~m}$, fue de $-0.65^{\circ} \mathrm{C}$ (Wilcke et al., 2008); entre $0 \mathrm{~m}$ y $2249 \mathrm{~m}$, fue de $-0.56{ }^{\circ} \mathrm{C}$ (Rolland, 2003); entre $600 \mathrm{~m}$ y $1400 \mathrm{~m}$ fue de $-0.52{ }^{\circ} \mathrm{C}$ (Wang, Chou, Chiou, Tian y Chiu, 2016); y entre $400 \mathrm{~m}$ y $1000 \mathrm{~m}$ fue de $-0.44{ }^{\circ} \mathrm{C}$. Lo anterior denota influencia de las bajas temperaturas en la diferenciación de la vegetación (Blum, Roderjan y Galvão, 2011).

Los bosques tropicales son más sensibles a climas de mayores altitudes (Kharal, Meilby, Rayamajhi, Bhuju y Thapa, 2015). En gradientes altitudinales de $2700 \mathrm{~m}$ a 3300 $\mathrm{m}$ (Kharal et al., 2015), de $2865 \mathrm{~m}$ a $3550 \mathrm{~m}$ (Wang et al., 2014) y de $600 \mathrm{~m}$ a $1650 \mathrm{~m}$ (Sidor, Popa, Vlad y Cherubini, 2015), determinaron que los crecimientos de Abies spectabilis (D. Don) Spach, Sabina preewalskii Kom. y Picea abies (L.) Karst. tuvieron correlación negativa con la TMA y correlación positiva con la PA, respectivamente. En el gradiente altitudinal de $100 \mathrm{~m}$ a $2200 \mathrm{~m}$, el incremento de la diversidad de plantas dependió de la precipitación de la estación cálida, siendo limitante la estación seca (MartínezCamilo, González-Espinosa, Ramírez-Marcial, Cayuela y Pérez-Farrera, 2018). En estación seca, las plantas hacen ajustes hidráulicos para un uso conservador del agua debido a su baja disponibilidad, además existe déficit de presión de vapor y de radiación solar debido al aumento de la altitud (Rosado, Joly, Burgess, Oliveira y Aidar, 2015). Las condiciones estacionales adversas actúan como filtro climático, lo cual favorece que prosperen especies más específicas con mayor rusticidad, en consecuencia, influyen en la diversidad de las especies tropicales.

\section{El pH del suelo}

$\mathrm{El} \mathrm{pH}$ de los suelos de los bosques tropicales distribuidos en gradiente altitudinal (Fig. 2a), en general, son ácidos con valores que varían de 3.7 a 6.0. Los valores de $\mathrm{pH}$ disminuyen con el aumento de la altitud (Wilcke et al., 2008; Debnath et al., 2012; Martinson, Corre y Veldkamp, 2013; Whitaker et al., 2014; Matson, Corre, Burneo y Veldkamp, 2015; Camenzind et al., 2015; Malhi et al., 2017; Bahar et al., 2017; Nottingham et al., 2018; Hicks et al., 2019; Ding, Zang, Lu, Huang y Xu, 2019; De la Cruz-Amo et al., 2020; Llambí et al., 2020; Oliveras et al., 2020); no obstante, otros resultados muestran que los valores del $\mathrm{pH}$ de los suelos aumentan con el aumento de la altitud (Wang et al., 2016; He, Hou, Liu y Wen, 2016; Chen, Yu, González, Zou y Gao, 2017), el aumento del pH en estos casos posiblemente esté relacionado al material parental del suelo. En el gradiente altitudinal de $871 \mathrm{~m}$ a $4550 \mathrm{~m}$, el pH del suelo disminuye hasta $3000 \mathrm{~m}$, mientras que, a mayores altitudes de $3000 \mathrm{~m}$, el pH aumenta (Peters et al., 2019); posiblemente porque a altitudes superiores a $3000 \mathrm{~m}$ disminuye la PA, como se observa en la fig $1 \mathrm{~b}$. Esto permite inferir que se produce menor lixiviación de las bases del suelo por infiltración o percolación, además, las bajas TMA disminuyen la generación de ácidos orgánicos, reflejándose en un aumento del pH en el suelo sobre los $3000 \mathrm{~m}$ de altitud (Fig. 2a). 
TABLA 1. Gradiente altitudinal, temperatura media anual (TMA), precipitación anual (PA), potencial de hidrogeniones (pH), nitrógeno total (NT), fósforo total (PT), carbono total $(\mathrm{CT})$, relación carbono/nitrógeno $(\mathrm{C} / \mathrm{N})$ en el suelo a diferentes altitudes y latitudes en bosques tropicales.

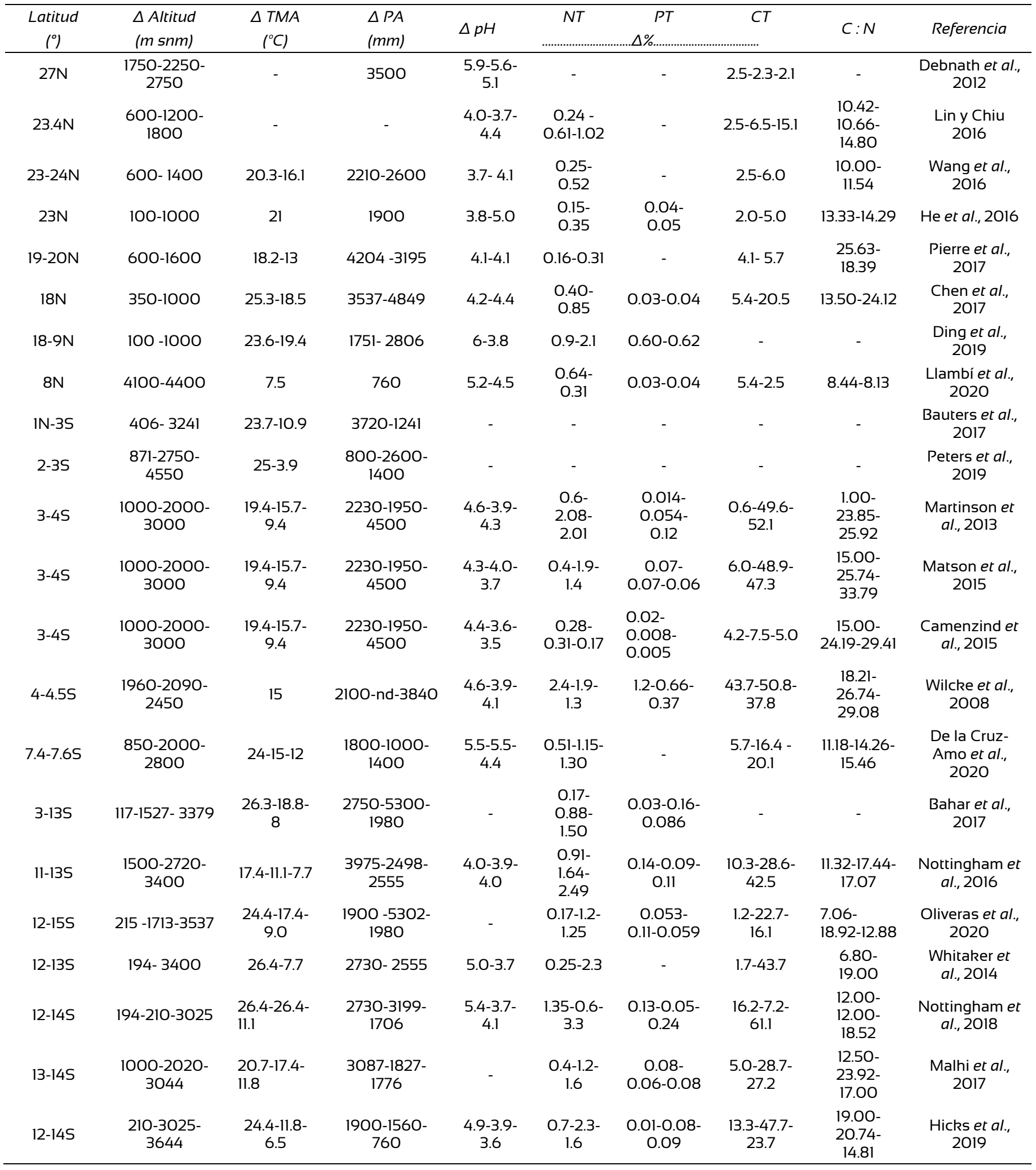



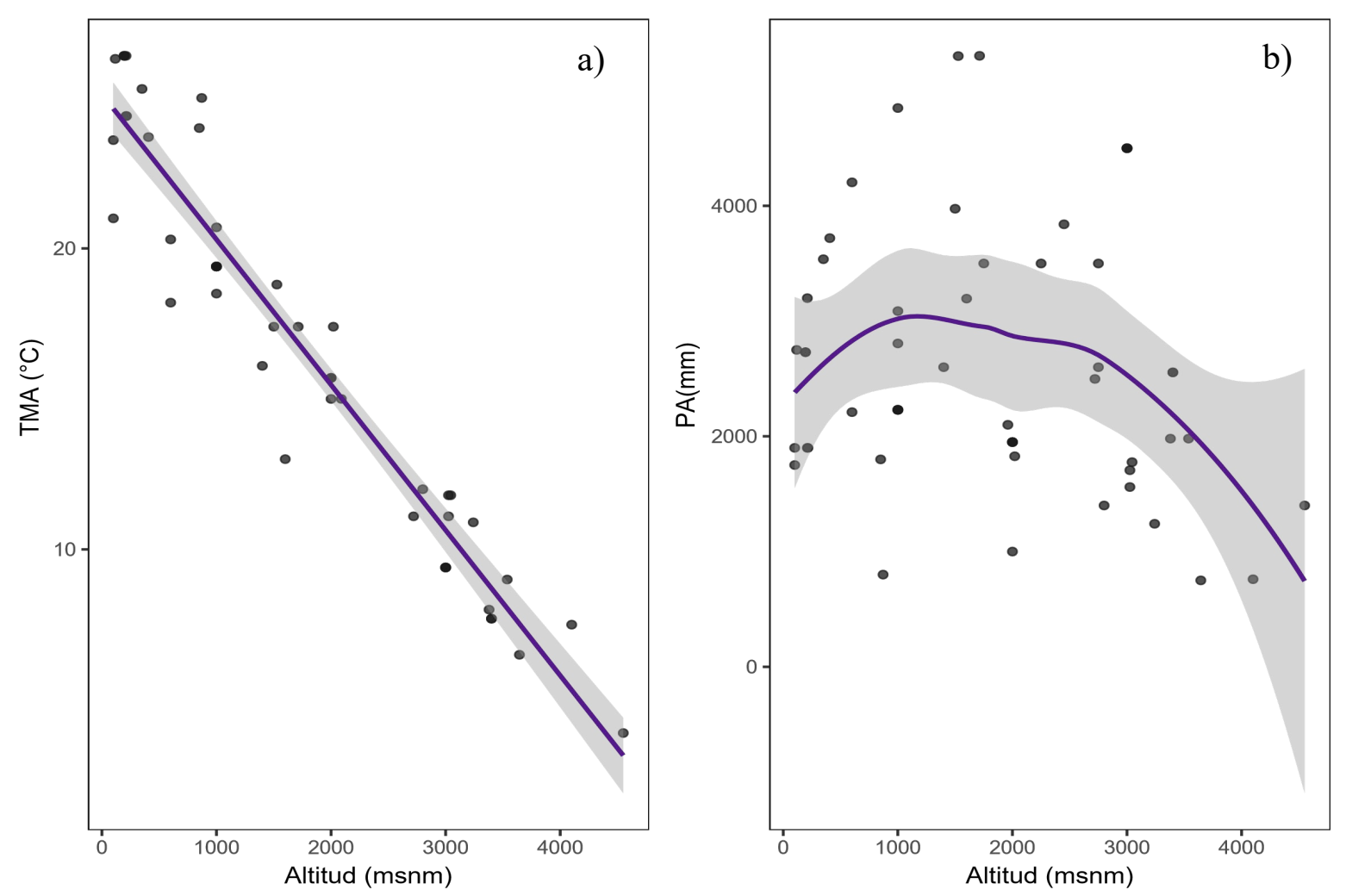

FIGURA 1. Gradiente altitudinal desde $0 \mathrm{~m}$ hasta más de $4000 \mathrm{~m}$. a) temperatura media anual (TMA) y b) precipitación anual (PA) en bosque tropical.

\section{Fertilidad del suelo}

En los gradientes altitudinales, los nutrientes del suelo presentan una relación inversa con la altitud (Soethe, Wilcke, Homeier, Lehmann y Engels, 2008; Wilcke et al., 2008). Cuando el gradiente altitudinal sobrepasa los 3000 $\mathrm{m}$, el nitrógeno $(\mathrm{N})$ asimilable en el suelo disminuye (Fahey, Sherman y Tanner, 2015; Ding et al., 2019;) por la baja mineralización del $\mathrm{N}$ debido a las bajas temperaturas y el aumento de la humedad del suelo (Fahey et al., 2015; Malhi et al., 2017); pues la humedad del suelo se incrementa con el aumento de la altitud (Chen et al., 2017; Ding et al., 2019; Hicks et al., 2019; Llambí et al., 2020). El N total en el suelo, aumenta a razón de $4 \mathrm{Mg} \mathrm{ha-1}$ por cada $1000 \mathrm{~m}$ de incremento de altitud (Ma y Chang, 2019). En la fig. 2b, se puede observar el comportamiento creciente del $\mathrm{N}$ total con el aumento de la altitud, con máximos hasta 3000 m; a partir de esta altitud, empieza a disminuir. Estos datos del $\mathrm{N}$ total corresponden a diferentes investigaciones en bosques tropicales (Martinson et al., 2013; Matson et al.,
2015; Baldos et al., 2015; Camenzind et al., 2015; Lin y Chiu 2016; Wang et al., 2016; He et al., 2016; Malhi et al., 2017; Pierre et al., 2017; Chen et al., 2017; Bahar et al., 2017; Hicks et al., 2019; Oliveras et al., 2020; De la Cruz-Amo et al., 2020), con valores mínimos de $0.15 \%$ a $0.17 \%$ entre $100 \mathrm{~m}$ y 600 m (He et al., 2016; Pierre et al., 2017;) y máximos de $2.5 \%$ a $3.3 \%$ entre $3000 \mathrm{~m}$ y $3400 \mathrm{~m}$ (Nottingham et al., 2018; Nottingham et al., 2016); a excepción de Wilcke et al. (2008) que determinaron que el porcentaje de $\mathrm{N}$ total disminuye con el aumento de la altitud. Cuando los gradientes altitudinales sobrepasan altitudes de $3000 \mathrm{~m}$, el porcentaje de $\mathrm{N}$ total disminuye juntamente con la producción vegetal (Martinson et al., 2013; Camenzind et al., 2015; Llambí et al., 2020). En ese sentido, a mayor altitud, menor es la concentración del $\mathrm{N}$ atmosférico $\mathrm{y}$, en consecuencia, es menor aporte de $\mathrm{N}$ atmosférico al suelo por precipitación pluvial. Lo anterior disminuye la fijación biológica y al mismo tiempo disminuye la producción vegetal. 

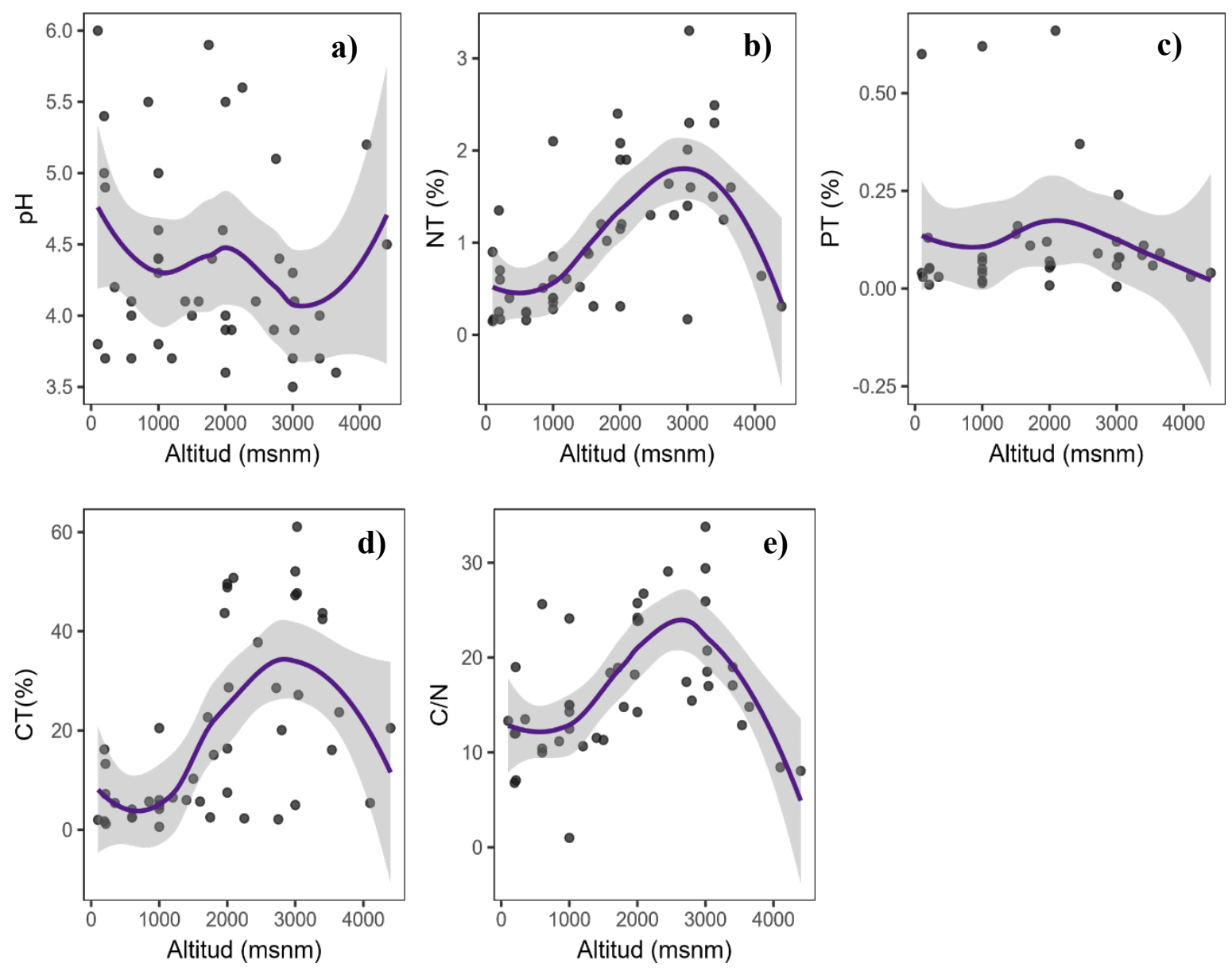

FIgurA 2. Gradiente altitudinal desde $0 \mathrm{~m}$ hasta más de $4000 \mathrm{~m}$. a) potencial de hidrogeniones (pH), b) nitrógeno total $(\mathrm{NT}), \mathrm{c})$ fósforo total $(\mathrm{PT}), \mathrm{d})$ carbono total $(\mathrm{CT})$, e) relación carbono/nitrógeno $(\mathrm{C} / \mathrm{N})$.

Con relación al fosforo total (PT) en gradientes altitudinales, no se observó una tendencia clara con incremento de la altitud (Fig. 2c). Investigaciones de Martinson et al. (2013), He et al. (2016), Chen et al. (2017), Nottingham et al. (2018), Ding et al. (2019), Hicks et al. (2019) y Oliveras et al. (2020) determinaron una relación directa entre la variación de la altitud con el aumento de los valores del P. Entretanto, otros estudios determinaron que los valores de $\mathrm{P}$ disminuyen con el aumento de la altitud (Wilcke et al., 2008; Camenzind et al., 2015; Matson et al., 2015; Nottingham et al., 2016). La capacidad de absorción del $\mathrm{P}$ por las plantas disminuye de $0.9 \mathrm{mg} \mathrm{kg}^{-1}$ a $0.2 \mathrm{mg} \mathrm{kg}$ ${ }^{1}$ cuando aumenta la altitud de $10 \mathrm{~m}$ a $1000 \mathrm{~m}$ (He et al., 2016), a pesar de que el P asimilable se incrementa con el aumento de la altitud (Ding et al., 2019).
Con respecto a $\mathrm{K}, \mathrm{Ca}, \mathrm{Fe}, \mathrm{Al}$ y $\mathrm{Mg}$ en el suelo, los estudios indican que estos elementos disminuyen con el aumento de la altitud en el gradiente altitudinal de bosques tropicales (Fig. 3). El K disminuyó de $0.23 \%$ a $0.01 \%$ entre 1000 m y 3000 m (Camenzind et al., 2015); de 0.31\% a $0.11 \%$ entre $1960 \mathrm{~m}$ y $2450 \mathrm{~m}$ (Wilcke et al., 2008). El Ca disminuyó de $0.6 \mathrm{mg} \mathrm{g}^{-1}$ a $0.2 \mathrm{mg} \mathrm{g}^{-1}$ entre $350 \mathrm{~m}$ y $1000 \mathrm{~m}$ (Chen et al., 2017); y de $0.38 \mathrm{mg} \mathrm{g}^{-1}$ a $0.10 \mathrm{mg} \mathrm{g}^{-1}$ y a 0.14 $\mathrm{mg} \mathrm{g}^{-1}$ entre $1000 \mathrm{~m}, 2000 \mathrm{~m}$ y $3000 \mathrm{~m}$ (Camenzind et al., 2015); y de $3.6 \mathrm{mg} \mathrm{g}^{-1}$ a $0.18 \mathrm{mg} \mathrm{g}^{-1}$ entre $1960 \mathrm{~m}$ y $2450 \mathrm{~m}$ (Wilcke et al., 2008). El Fe disminuyó de $12.1 \mathrm{mg} \mathrm{g}{ }^{-1}, 0.2 \mathrm{mg}$ $\mathrm{g}^{-1}$ y $0.1 \mathrm{mg} \mathrm{g}^{-1}$; el Al disminuyó de $40.3 \mathrm{mg} \mathrm{g}^{-1}, 0.8 \mathrm{mg} \mathrm{g}^{-1} \mathrm{y}$ $0.4 \mathrm{mg} \mathrm{g}^{-1}$; y el Mg disminuyó de $0.93 \mathrm{mg} \mathrm{g}^{-1}, 0.12 \mathrm{mg} \mathrm{g}^{-1} \mathrm{y}$ $0.09 \mathrm{mg} \mathrm{g}^{-1}$, entre $1000 \mathrm{~m}, 2000 \mathrm{~m}$ y $3000 \mathrm{~m}$, respectivamente (Camenzind et al., 2015). La tendencia de 
$\mathrm{m}$

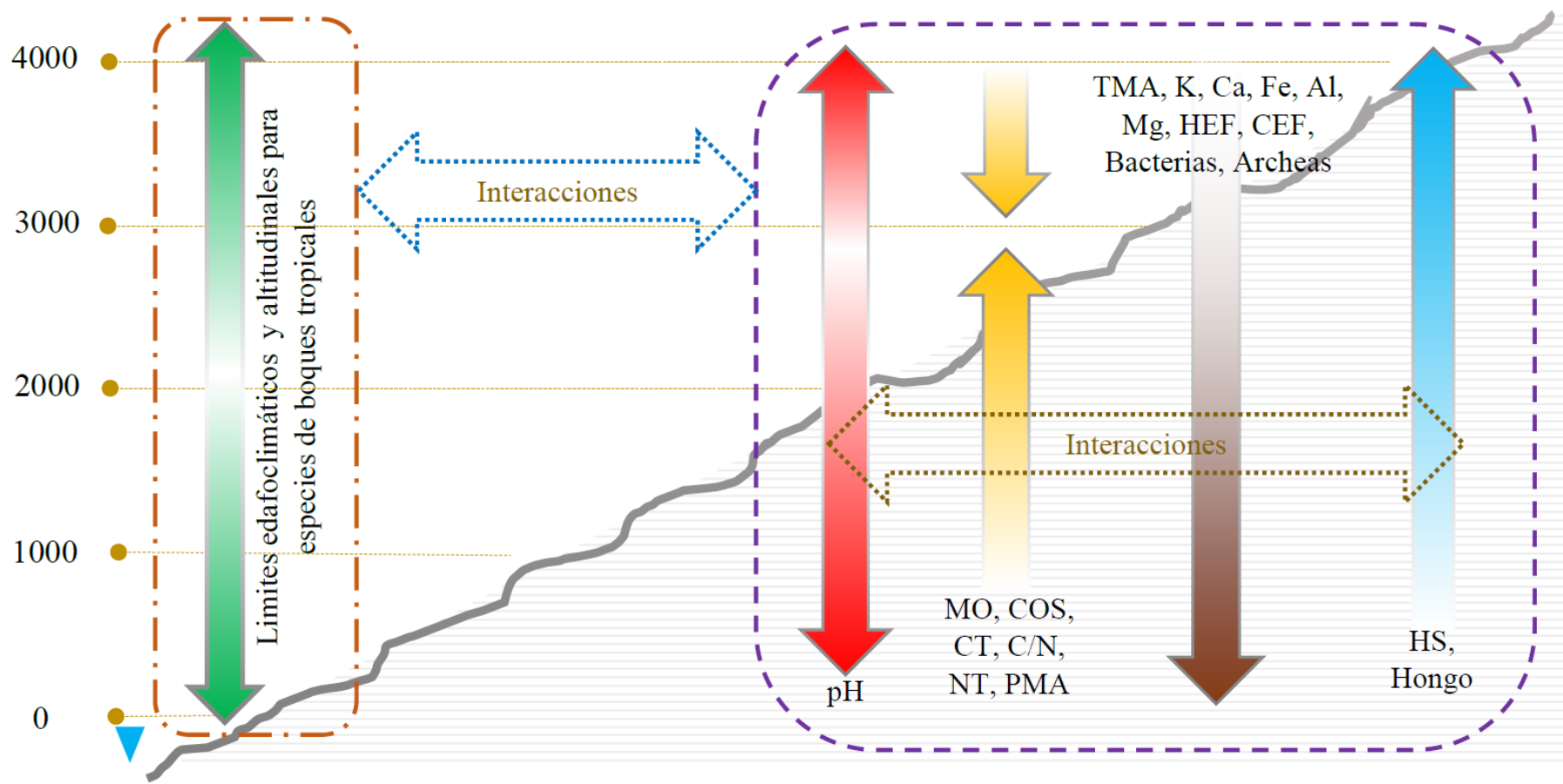

FIgURA 3. Perfil de gradiente altitudinal desde $0 \mathrm{~m}$ hasta más de $4000 \mathrm{~m}$ que representa el comportamiento de la temperatura media anual (TMA) y precipitación anual (PA), humedad de suelo (HS), potencial de hidrogeniones (pH), contenido total de carbono (CT), fosforo total $(\mathrm{PT})$, nitrógeno total $(\mathrm{NT})$, calcio $(\mathrm{Ca})$, potasio $(\mathrm{K})$, fierro $(\mathrm{Fe})$, aluminio $(\mathrm{Al})$, magnesio $(\mathrm{Mg})$, relación carbono/ nitrógeno $(\mathrm{C} / \mathrm{N})$, altura de especies forestales (HEF), crecimiento de especies forestales(CEF), hongo, bacterias y archaeas en el suelo en bosques tropicales.

disminución de los nutrientes en el suelo se asociada con las bajas TMA, mayor humedad del suelo y altas concentraciones de compuestos fenólicos que ralentizan la descomposición y mineralización de la metería orgánica en gradientes altitudinales más altos (Wilcke et al., 2008). Haciendo estas consideraciones se deduce que las especies forestales a mayor altitud están sometidas a complejos límites edafoclimáticos y altitudinales (Fig. 3), lo cual favorece que prosperen especies rústicas o endémicas, con disminución de la diversidad.

\section{Carbono orgánico en el suelo}

La profundidad del suelo es mayor en altitudes inferiores, contrario a los horizontes orgánicos que aumentan con la altitud de los gradientes altitudinales de los bosques tropicales (Soethe et al., 2008); tal es el caso, para $1000 \mathrm{~m}$,
$2000 \mathrm{~m}$ y $3000 \mathrm{~m}$ fueron $0.30 \mathrm{~cm}$ y $40.0 \mathrm{~cm}$ (Martinson $e t$ al., 2013) y para $194 \mathrm{~m}, 210 \mathrm{~m}$ y $3025 \mathrm{~m}$ fueron $1 \mathrm{~cm}, 2 \mathrm{~cm}$ y $23.0 \mathrm{~cm}$ (Nottingham et al., 2018), respectivamente. Cuando se trata de la materia orgánica en el suelo, para 350 m y $1000 \mathrm{~m}$ se obtuvieron $22.5 \%$ y $48.2 \%$ (Chen et al., 2017); por lo mismo, el carbono orgánico aumenta a razón de $54.0 \mathrm{Mg} \mathrm{ha}^{-1}$ por cada $1000 \mathrm{~m}$ de incremento de altitud (Ma et al., 2019), en este sentido, para evaluaciones entre 194 m y $3025 \mathrm{~m}$, aumentó de $3.5 \mathrm{Mg} \mathrm{ha}^{-1}$ a $59.6 \mathrm{Mg} \mathrm{ha}^{-1}$ (Girardin et al., 2010); entre 406 m y 3217 m, aumentó de $96 \mathrm{Mg} \mathrm{ha}{ }^{-1}$ a $161 \mathrm{Mg} \mathrm{ha}^{-1}$ (Bauters et al., 2017) respectivamente. Del mismo modo, el porcentaje de CT en el suelo también se incrementa con el aumento de la altitud, sin embargo, a partir de los $3000 \mathrm{~m}$ estos valores disminuyen (Fig. 2d). El aumento de CT corresponde a investigaciones realizadas a altitudes inferiores a $3000 \mathrm{~m}$ 
(Wilcke et al., 2008a; Debnath et al., 2012; Martinson et al., 2013; Whitaker et al., 2014; Matson et al., 2015; Camenzind et al., 2015; Lin et al., 2016; Wang et al., 2016; Nottingham et al., 2016; He et al., 2016; Malhi et al., 2017; Pierre et al., 2017; Chen et al., 2017; Nottingham et al., 2018; Hicks et al., 2019; Oliveras et al., 2020; De la Cruz-Amo et al., 2020). El aumento del grosor de los horizontes orgánicos aumenta la materia orgánica y el porcentaje de CT en el suelo en el gradiente altitudinal entre $0 \mathrm{~m}$ y $3000 \mathrm{~m}$ (Fig. 2d, Fig. 3), esto se debe principalmente a la baja transformación del carbono orgánico en carbono mineral. La mineralización de la materia orgánica disminuye con el aumento de la altitud por la influencia de las bajas temperaturas (Wang et al., 2016) y por la saturación de agua en el suelo (proceso hidromórfico), que impide la migración descendente de la materia orgánica hacia horizontes inferiores (proceso podzolico). Lo anterior redunda en una baja mineralización y produce mayor concentración de carbono orgánico en el suelo (De la Cruz-Amo et al., 2020). Por esta razón, la agregación del suelo se incrementa con el aumento de la altitud, por el aumento de carbono orgánico en los macro agregados del suelo (Li et al., 2016).

Si los gradientes altitudinales sobrepasan los $3000 \mathrm{~m}$, el grosor de los horizontes orgánicos disminuyen progresivamente en el suelo, del mismo modo disminuye el CT sobre esta altitud (Fig. 2d). Los horizontes orgánicos en $194 \mathrm{~m}, 3025 \mathrm{~m}$ y $3400 \mathrm{~m}$ fueron de $0.7 \mathrm{~cm}, 22.8 \mathrm{~cm}$ y 14.0 cm (Whitaker et al., 2014); en 1776 m, 2020 m y 3537 m, fueron de $32 \mathrm{~cm}, 80 \mathrm{~cm}$ y $36 \mathrm{~cm}$ (Malhi et al., 2017); en 210 $\mathrm{m}, 3025 \mathrm{~m}$ y $3644 \mathrm{~m}$ fueron de $1.5 \mathrm{~cm}, 13.0 \mathrm{~cm}$ y $2.7 \mathrm{~cm}$ (Hicks et al., 2019), respectivamente. En estas altitudes también disminuye la materia orgánica en suelo con el aumento de la altitud, como lo confirman Llambí et al. (2020) al obtener 19.3\% y 9.5\% de mataría orgánica en 4100 m y 4600 m, respectivamente. La disminución del grosor de los horizontes orgánicos y del porcentaje de materia orgánica en el suelo en gradientes altitudinales superiores a 3000 m aluden a una baja producción vegetal, ocasionando por una menor dotación de agua de PA, retraso en los procesos bioquímicos por las bajas TMA (Fig. 1a y b, Fig. $2 \mathrm{~b}$ y d) y baja disponibilidad de nutrientes.

\section{Relación $\mathrm{C} / \mathrm{N}$ en el suelo}

El comportamiento de la relación $\mathrm{C} / \mathrm{N}$ depende del CT y NT, que en el gradiente altitudinal en altitudes entre $0 \mathrm{~m} \mathrm{y}$ $3000 \mathrm{~m}$ se incrementa con el aumento de la altitud, sin embargo, sobre esta altitud disminuye (Fig. 2e). El aumento entre $0 \mathrm{~m}$ y $3000 \mathrm{~m}$ y la disminución sobre los $3000 \mathrm{~m}$ de altitud de la relación $\mathrm{C} / \mathrm{N}$ corresponden a diferentes investigaciones (Wilcke et al., 2008; Martinson et al., 2013; Whitaker et al., 2014; Matson et al., 2015; Camenzind et al., 2015; Lin et al., 2016; Wang et al., 2016; He et al., 2016; Nottingham et al., 2016; Chen et al., 2017; Malhi et al., 2017; Nottingham et al., 2018; Hicks et al., 2019; Peters et al., 2019; Oliveras et al., 2020; De la Cruz-Amo et al., 2020; Llambí et al., 2020. ), a excepción de Pierre et al. (2017), quienes determinaron que, en un gradiente altitudinal de $600 \mathrm{~m}$ a $1600 \mathrm{~m}$, la relación $\mathrm{C} / \mathrm{N}$ disminuye con valores de 25.63 y 18.39 respectivamente. En un gradiente altitudinal de 1000 m a $3000 \mathrm{~m}$, la descomposición de la materia orgánica es mayor a menores altitudes, debido a la mayor presencia de microrganismos favorecidos por la baja relación de $\mathrm{C} / \mathrm{N}$ (Baldos et al., 2015). En consecuencia, se puede afirmar que a menores valores de la relación $\mathrm{C} / \mathrm{N}$, mayor es la descomposición de la materia orgánica en el suelo, por lo tanto, existe mayor disponibilidad de nutrientes para las plantas, lo que origina suelos fértiles y profundos con mayor diversidad de flora y fauna en altitudes inferiores; ocurre lo contrario en altitudes superiores (Fig. 2e).

\section{Hongos, bacterias y archaeas en el suelo}

En gradientes altitudinales, entre 871 m y 4550 m, en áreas naturales y de uso antrópico, los vegetales y animales disminuyen acentuadamente por la influencia de la altitud y la intensidad de uso de la tierra; sin embargo, los microrganismos no son afectados significativamente (Peters et al., 2019). En los gradientes altitudinales, los hongos, la baterías y las archaeas son afectados de manera diferente por la PA, la TMA, el pH, el carbono, el $\mathrm{N}$ y el P en el suelo. La riqueza de los hongos aumenta con el incremento de la altitud, de la PA y del contenido de carbono orgánico (Whitaker et al., 2014); pero, disminuye 
con el incremento del pH y el N (Pierre et al., 2017). La riqueza de bacterias aumenta con el incremento del $\mathrm{pH}$ y del carbono orgánico, pero disminuye con el incremento de la altitud, la PA y la relación C/P (Lin et al., 2016; Pierre et al., 2017). Según el tipo de horizontes del suelo, los hongos se encuentran en menor cantidad en los horizontes inorgánicos, las bacterias en los horizontes orgánicos y viceversa, respectivamente (Nottingham et al., 2016). La riqueza de archaeas aumenta con el incremento de la tasa de mineralización del N, por el aumento de la TMA en bajas altitudes (Pierre et al., 2017), disminuyendo el ciclo del $\mathrm{N}$ en el suelo (Baldos et al., 2015). Por ello, es posible inferir que la riqueza de las archaeas disminuye con la altitud. Cuando se trata de la diversidad de hongos y bacterias, esta disminuye con el aumento de la altitud en gradiente altitudinal de $200 \mathrm{~m}$ a $3400 \mathrm{~m}$ (Nottingham et al., 2018; Nottingham et al., 2016); mientras que, en gradiente altitudinal de $3000 \mathrm{~m}$ a $3900 \mathrm{~m}$, el pH en el suelo aumenta, incrementando la diversidad de los hongos (Liu, Liu, Chen, Wang y Zhang, 2018).

En altitudes de 1000 m, 2000 m y 3000 m, en suelos fertilizados con $\mathrm{N}$ y $\mathrm{P}$, se obtuvo aumento significativo de hongos por la influencia del P (Camenzind et al., 2015; Camenzind, Scheu y Rillig, 2019); a mayores altitudes, el P aumentó el tamaño de las hifas; comportamiento contrario ocasionó el N (Camenzind et al., 2019). Por otro lado, cuando al suelo se fertiliza con N, P, K y Na, en altitudes de 194 m, 210 m y 3025 m, se obtuvo aumento de hongos en restos de celulosa, por la adición de $\mathrm{N}$, lo que sugiere que la principal limitante para degradación de la celulosa es la falta de $\mathrm{N}$ en el suelo (Nottingham et al., 2018). Estos resultados destacan la importancia de la relación de los microrganismos con el $\mathrm{N}$ y el $\mathrm{P}$ en la descomposición de la materia orgánica en el suelo en gradientes altitudinales.

\section{Estructura poblacional de los bosques}

En diferentes gradientes altitudinales, Kharal et al. (2015), entre $2300 \mathrm{~m}$ y $2700 \mathrm{~m}$, obtuvieron mayor área basal de $A$. spectabilis (D. Don) Spach en altitudes medias; Wilcke et al. (2008), entre 1960 m y 2450 m, determinaron que el área basal de los árboles disminuye con el aumento de la altitud; en contraste, Bauters et al. (2017), entre 406 m y 3241 m, determinaron que el área basal es favorecida por el aumento de la altitud. En cuanto a la riqueza de los árboles en gradiente altitudinal, Acharya, Chettri y Vijayan (2011), entre $300 \mathrm{~m}$ y $4700 \mathrm{~m}$, determinaron que la riqueza de los árboles tuvo correlación positiva con la TMA, la humedad relativa, la PA y la evapotranspiración; Zhu et al. (2019); entre $281 \mathrm{~m}$ y $1175 \mathrm{~m}$ determinaron que la riqueza de los árboles presenta valores máximos en altitudes medias. Por su parte, Desalegn y Beierkuhnlein (2010), entre $1200 \mathrm{~m}$ y $2700 \mathrm{~m}$, obtuvieron mayor riqueza de árboles a mayor altitud; sin embargo, Ding et al. (2019), entre $100 \mathrm{~m}$ y 1000 $\mathrm{m}$, y Peters et al. (2019), entre $871 \mathrm{~m}$ y $4550 \mathrm{~m}$, determinaron que la riqueza de los árboles decrece significativamente con la altitud. Los diferentes datos en cuanto a riqueza y área basal de los árboles se pueden asociar a las diferentes condiciones edafoclimáticas, edades de árboles, amplitud de altitud y latitud (Tabla 1); esta última hace variar el fotoperiodo, en consecuencia, la capacidad fotosintética de las plantas, afectando la estructura de los árboles. No obstante, con uso antrópico, entre $871 \mathrm{~m}$ y $4550 \mathrm{~m}$, la riqueza de las plantas disminuye por la intensidad de uso de la tierra, siendo las zonas áridas las que más pierden, con hasta 50\% (Peters et al., 2019).

De acuerdo con Gairola, Rawal y Todaria (2008), entre $2800 \mathrm{~m}$ y $3600 \mathrm{~m}$, la densidad de los árboles disminuye acentuadamente con el aumento de la altitud; en contraste con los resultados de Bauters et al. (2017), entre $406 \mathrm{~m} \mathrm{y}$ $3241 \mathrm{~m}$, y de Martinson et al. (2013), entre $1000 \mathrm{~m}$ y 3000 $\mathrm{m}$, quienes determinaron que la densidad de los árboles aumenta con la altitud. Por su parte, González-Rojas, Murillo-Cruz, Virginio-Filho y Ávila-Arias (2018) determinaron que la mayor densidad de los árboles disminuye su crecimiento. Se puede inferir que la disminución de crecimiento por mayor densidad de árboles se debe a la competencia por luz, agua y nutrientes entre plantas, independientemente del gradiente altitudinal.

Cuando se trata de la diversidad de plantas, estas disminuyen con el aumento de la altitud en gradiente altitudinal de $200 \mathrm{~m}$ a $3400 \mathrm{~m}$ (Nottingham et al., 2016; 
Nottingham et al., 2018). Según Brambach, Leuschner y Culmsee (2017), entre $1000 \mathrm{~m}$ y $2500 \mathrm{~m}$, y Peters et al. (2019), entre $871 \mathrm{~m}$ y $4550 \mathrm{~m}$, determinaron que familias, géneros y especies disminuyen con la altitud; comportamiento contrario presentan las especies endémicas que aumentan con la altitud. Por su parte, Asner et al. (2017) y Oliveras et al. (2020), en gradientes altitudinales entre $215 \mathrm{~m}$ y $3537 \mathrm{~m}$ determinaron que familias, géneros y especies de las plantas presentan un comportamiento unimodal. Estos estudios sugieren que familias, géneros y especies de plantas presentan límites altitudinales y climáticos, de diferente amplitud, y que fuera de estos no prosperan satisfactoriamente. En este sentido, es probable que la disminución o el aumento de las familias, géneros y especies de plantas se deban a que las evaluaciones se hicieron en una fracción y no en toda la amplitud climática y altitudinal donde prosperan.

\section{Respuestas fisiológicas de los bosques a los gradientes altitudinales}

El crecimiento anual de las especies forestales en los trópicos es afectado negativamente por el aumento de la altitud en gradientes altitudinales (Gairola et al., 2008; Wilcke et al., 2008; Martinson et al., 2013; Fahey et al., 2015; Asner et al., 2017; Bauters et al., 2017; González-Rojas et al., 2018; De la Cruz-Amo et al., 2020); a razón de que el período de crecimiento anual es menor a mayor altitud, retrasándose de 3 días a 4 días cada $100 \mathrm{~m}$ de incremento de altitud, o aumentado en 7 días cada $1{ }^{\circ} \mathrm{C}$ de aumento de la temperatura (Wang et al., 2014). Por consiguiente, a mayor altitud se encuentran árboles pequeños con múltiples troncos, generalmente torcidos, con alta relación del diámetro de tronco y altura de copa (Fahey et al., 2015) y con madera de baja densidad (De la Cruz-Amo et al., 2020). Estas características se deben a la poca luz disponible, por la alta saturación de nubes que limita la fotosíntesis de las plantas (Fahey et al., 2015). Pese a la baja disponibilidad de luz, la mayor capacidad fotosintética de las plantas ocurre a mayor altitud (Bahar et al., 2017; Malhi et al., 2017) debido a que presentan mayor eficiencia de uso del $\mathrm{N}$ por unidad de área foliar (Bahar et al., 2017).

De acuerdo con Bahar et al., 2017; Asner et al., 2017; Llambí et al., 2020, en las hojas de las plantas, la masa foliar, el contenido de $\mathrm{P}$ y el de $\mathrm{N}$ aumentan con la altitud; eto es contrario a lo registrado por Bauters et al. (2017) y Ding et al. (2019). Es probable que estos casos se deban a la baja disponibilidad de estos nutrientes en el suelo por el aumento de la altitud (Fig. 3) o, en su defecto, están disponibles, pero no en su forma asimilable para las plantas, como es el caso del N. Esto es debido a las bajas TMA y mayor HS por efecto del aumento de la altitud (Wilcke et al., 2008; Fahey et al., 2015; Ding et al., 2019).

La disponibilidad de nutrientes en el suelo es proporcional al contenido de nutrientes en las hojas de las plantas. En este sentido, el contenido de Ca y K en el suelo disminuye con el aumento de la altitud (Wilcke et al. 2008; Camenzind et al., 2015); del mismo modo, con el aumento de la altitud, disminuye el contenido de $\mathrm{K}$ y $\mathrm{Ca}$ en las hojas de especies forestales (Asner et al., 2016; Asner et al., 2017; Bauters et al., 2017). También disminuyen con el aumento de la altitud las relaciones N/P, N/C en las hojas, el índice de área foliar (Bauters et al., 2017; Malhi et al., 2017; Ding et al., 2019), la productividad forestal y la biomasa (Malhi et al., 2017).

\section{CONCLUSIONES}

Los bosques tropicales se encuentran condicionados por las propiedades del suelo, así como por factores y elementos climáticos que, cuando interactúan, generan límites espaciales y temporales para las especies forestales, que poco son evidenciados cuando los estudios se hacen a escala local. El factor climático de mayor importancia en los bosques tropicales son los gradientes altitudinales, que se encuentran desde $0 \mathrm{~m}$ a más de $3500 \mathrm{~m}$, haciendo variar notablemente los elementos climáticos como temperatura y precipitación y, en el suelo, propiedades físicas, químicas y biológicas, como el $\mathrm{pH}$, los nutrientes, el contenido de carbono y los microrganismos, entre otros. En consecuencia, hacen variar las respuestas fisiológicas de las especies forestales, afectando su estructura poblacional. Es 
necesario analizar y considerar la influencia edafoclimática y altitudinal en los bosques forestales para su gestión; apremia abordar mayores estudios para una mejor comprensión de la interacción de los bosques tropicales con el medio ambiente, con fines de uso de la tierra y de conservación.

\section{RECONOCIMIENTOS}

El primer autor agradece a la Coordenação de Aperfeiçoamento de Pessoal de Ensino Superior (Capes) de Brasil que, mediante el Programa de Alianzas para la Educación y la Capacitación (PAEC) de la Organización de los Estados Americanos (OEA), financió esta investigación, como parte del programa de doctorado en Agricultura Tropical de la Universidade Federal de Mato Grosso (UFMT).

\section{REFERENCIAS}

Acharya, B. K., Chettri, B., \& Vijayan, L. (2011). Distribution pattern of trees along an elevation gradient of Eastern Himalaya, India. Acta Oecologica, 37(4), 329-336. doi: 10.1016/j.actao.2011.03.005

Asner, G. P., \& Martin, R. E. (2016). Convergent elevation trends in canopy chemical traits of tropical forests. Global Change Biology, 22(6), 2216-2227. doi: 10.1111/gcb.13164

Asner, G. P., Martin, R. E., Anderson, C. B., Kryston, K., Vaughn, N., Knapp, D. E., ..., \& Tupayachi, R. (2017). Scale dependence of canopy trait distributions along a tropical forest elevation gradient. New Phytologist, 214(3), 973-988. doi: 10.1111/nph.14068

Aybar-Camacho, C.; Lavado-Casimiro, W.; Sabino, E.; Ramírez, S.; Huerta, J., \& Felipe-Obando, O. (2017). Atlas de zonas de vida del Perú - Guia Explicativa. Servicio Nacional de Meteorología e Hidrología del Perú (SENAMHI). Dirección de Hidrología. 30 p.

Bahar, N. H., Ishida, F. Y., Weerasinghe, L. K., Guerrieri, R., O'Sullivan, O. S., Bloomfield, K. J., ..., \& Phillips, O. L. (2017). Leaf-level photosynthetic capacity in lowland Amazonian and highelevation Andean tropical moist forests of Peru. New Phytologist, 214(3), 1002-1018. doi: 10.1111/nph.14079

Baldos, A. P., Corre, M. D., \& Veldkamp, E. (2015). Response of N cycling to nutrient inputs in forest soils across a 1000-3000 m elevation gradient in the Ecuadorian Andes. Ecology, 96(3), 749761. doi: 10.1890/14-0295.1

Bauters, M., Verbeeck, H., Demol, M., Bruneel, S., Taveirne, C., Van der Heyden, D., ..., \& Boeckx, P. (2017). Parallel functional and stoichiometric trait shifts in South American and African forest communities with elevation. Biogeosciences, 14(23), 5313-5321. doi: 10.5194/bg-14-5313-2017

Blum, C. T., Roderjan, C. V., \& Galvão, F. (2011). O clima e sua influência na distribuição da Floresta Ombrófila Densa na Serra da Prata, Morretes, Paraná. Floresta, 41(3), 589-598. doi: 10.5380/rf.v41i3.24052

Brambach, F., Leuschner, C., Tjoa, A., \& Culmsee, H. (2017). Diversity, endemism, and composition of tropical mountain forest communities in Sulawesi, Indonesia, in relation to elevation and soil properties. Perspectives in Plant Ecology, Evolution and Systematics, 27, 68-79. doi: 10.1016/j.ppees.2017.06.003

Camenzind, T., Homeier, J., Dietrich, K., Hempel, S., Hertel, D., Krohn, A., ..., \& Rillig, M. C. (2015). Opposing effects of nitrogen versus phosphorus additions on mycorrhizal fungal abundance along an elevational gradient in tropical montane forests. Soil Biology and Biochemistry, 94, 37-47. doi: 10.1016/j.soilbio.2015.11.011

Camenzind, T., Scheu, S., \& Rillig, M. C. (2019). Expanding the toolbox of nutrient limitation studies: A novel method of soil microbial in-growth bags to evaluate nutrient demands in tropical forests. Functional Ecology, 33(8), 1536-1548. doi: 10.1111/13652435.13352

Cardinael, R., Chevallier, T., Cambou, A., Beral, C., Barthès, B. G., Dupraz, C., ..., \& Chenu, C. (2017). Increased soil organic carbon stocks under agroforestry: a survey of six different sites in France. Agriculture, Ecosystems \& Environment, 236, 243-255. doi: 10.1016/j.agee.2016.12.011

Chen, D., Yu, M., González, G., Zou, X., \& Gao, Q. (2017). Climate impacts on soil carbon processes along an elevation gradient in the tropical luquillo experimental forest. Forests, 8(3), 90. doi: $10.3390 /$ f 8030090

De la Cruz-Amo L, Bañares-de-Dios G, Cala V, Granzow-de la Cerda I, Espinosa CI, Ledo A, Salinas N, Macía MJ and Cayuela L .(2020). Trade Offs Among Aboveground, Belowground, and Soil Organic Carbon Stocks Along Altitudinal Gradients in Andean Tropical Montane Forests. Frontiers in Plant Science. 11,106. doi: $10.3389 /$ fpls.2020.00106

Debnath, P., Deb, P., Sen, D., Pattannaik, S. K., Sah, D., \& Ghosh, S. K. (2012). Physico-chemical properties and its relationship with water holding capacity of cultivated soils along altitudinal gradient in Sikkim. International Journal of Agriculture, Environment and Biotechnology, 5(2), 161-166.

Desalegn, W., \& Beierkuhnlein, C. (2010). Plant species and growth form richness along altitudinal gradients in the southwest Ethiopian highlands. Journal of Vegetation Science, 21(4), 617-626. doi: 10.1111/j.1654-1103.2010.01177.x 
Ding, Y., Liu, G., Zang, R., Zhang, J., Lu, X., \& Huang, J. (2016). Distribution of vascular epiphytes along a tropical elevational gradient: disentangling abiotic and biotic determinants. Scientific Reports, 6, 19706. doi: 10.1038/srep19706

Ding, Y., Zang, R., Lu, X., Huang, J., \& Xu, Y. (2019). The effect of environmental filtering on variation in functional diversity along a tropical elevational gradient. Journal of Vegetation Science, 30(5), 973-983. doi: 10.1111 /jvs.12786

Fahey, T. J., Sherman, R. E., \& Tanner, E. V. J. (2015). Tropical montane cloud forest: environmental drivers of vegetation structure and ecosystem function. Journal of Tropical Ecology, 32(05), 355-367. doi: 10.1017/S0266467415000176

Gairola, S., Rawal, R. S., \& Todaria, N. P. (2008). Forest vegetation patterns along an altitudinal gradient in sub-alpine zone of west Himalaya, India. African Journal of Plant Science, 2(6), 42-48. doi: 10.5897 / AJPS. 9000126

Girardin, C. A. J., Malhi, Y., Aragao, L. E. O. C., Mamani, M., Huaraca Huasco, W., Durand, L., ..., \& Salinas, N. (2010). Net primary productivity allocation and cycling of carbon along a tropical forest elevational transect in the Peruvian Andes. Global Change Biology, 16(12), 3176-3192. doi: 10.1111/j.13652486.2010.02235.x

González-Rojas, M., Murillo-Cruz, R., D. M., Virginio-Filho, E., \& Ávila-Arias, C. (2018). Influence of biophysical and management factors on the growth of Cedrela odorata L. in association with coffee in Pérez Zeledón, Costa Rica. Revista Forestal Mesoamericana Kurú, 15(36), 46-58. doi: 10.18845/rfmk.v15i36.3420

He, X., Hou, E., Liu, Y., \& Wen, D. (2016). Altitudinal patterns and controls of plant and soil nutrient concentrations and stoichiometry in subtropical China. Scientific reports, 6, 24261. doi: $10.1038 /$ srep24261

Hicks, L. C., Meir, P., Nottingham, A. T., Reay, D. S., Stott, A. W., Salinas, N., \& Whitaker, J. (2019). Carbon and nitrogen inputs differentially affect priming of soil organic matter in tropical lowland and montane soils. Soil Biology and Biochemistry, 129, 212 222. doi: $10.1016 /$ j.soilbio.2018.10.015

Holdridge, L. R. (1967). Life zone ecology. Life zone ecology, Tropical Science Center. San Jose', Costa Rica, 206 p.

Kharal, D. K., Meilby, H., Rayamajhi, S., Bhuju, D., \& Thapa, U. K. (2015). Tree ring variability and climate response of Abies spectabilis along an elevation gradient in Mustang, Nepal. Banko Janakari, 24(1), 3-13. doi: 10.3126/banko.v24i1.13473

Li, L., Vogel, J., He, Z., Zou, X., Ruan, H., Huang, W., ..., \& Bianchi, T. S. (2016). Association of soil aggregation with the distribution and quality of organic carbon in soil along an elevation gradient on
Wuyi Mountain in China. PLoS ONE, 11(3). doi: 10.1371/journal.pone. 0150898

Lin, Y. T., \& Chiu, C. Y. (2016). Elevation gradient of soil bacterial communities in bamboo plantations. Botanical studies, 57(1),8. doi: 10.1186/s40529-016-0123-0

Liu, D., Liu, G., Chen, L., Wang, J., \& Zhang, L. (2018). Soil pH determines fungal diversity along an elevation gradient in Southwestern China. Science China Life Sciences, 61(6), 718-726. doi: 10.1007/s11427-017-9200-1

Llambí, L. D., Durbecq, A., Cáceres-Mago, K., Cáceres, A., Ramírez, L., Torres, E., \& Méndez, Z. (2020). Interactions between nurseplants and an exotic invader along a tropical alpine elevation gradient: growth-form matters. Alpine Botany, 1-15. doi: $10.1007 /$ s00035-020-00235-6

Ma, M., \& Chang, R. (2019). Temperature drive the altitudinal change in soil carbon and nitrogen of montane forests: Implication for global warming. Catena, 182, 104126. doi: 10.1016/j.catena.2019.104126

Malhi, Y., Girardin, C. A., Goldsmith, G. R., Doughty, C. E., Salinas, N., Metcalfe, D. B., ..., \& Aragão, L. E. (2017). The variation of productivity and its allocation along a tropical elevation gradient: a whole carbon budget perspective. New Phytologist, 214(3), 10191032. doi: $10.1111 /$ nph.14189

Martínez-Camilo, R., González-Espinosa, M., Ramírez-Marcial, N., Cayuela, L., \& Pérez-Farrera, M. Á. (2018). Tropical tree species diversity in a mountain system in southern Mexico: local and regional patterns and determinan factors. Biotropica, 50(3), 499509. doi: 10.1111/btp.12535

Martinson, G. O., Corre, M. D., \& Veldkamp, E. (2013). Responses of nitrous oxide fluxes and soil nitrogen cycling to nutrient additions in montane forests along an elevation gradient in southern Ecuador. Biogeochemistry, 112(1-3), 625-636. doi: 10.1007/s10533012-9753-9

Matson, A. L., Corre, M. D., Burneo, J. I., \& Veldkamp, E. (2015). Freeliving nitrogen fixation responds to elevated nutrient inputs in tropical montane forest floor and canopy soils of southern Ecuador. Biogeochemistry, 122(2-3), 281-294. doi: 10.1007/s10533014-0041-8

Nottingham, A. T., Hicks, L. C., Ccahuana, A. J., Salinas, N., Bååth, E., \& Meir, P. (2018). Nutrient limitations to bacterial and fungal growth during cellulose decomposition in tropical forest soils. Biology and fertility of soils, 54(2), 219-228. doi: 10.1007/s00374-017$1247-4$

Nottingham, A., Fierer, N., Turner, B., Whitaker, J., Ostle, N., McNamara, N., ..., \& Silman, M. (2016). Temperature drives plant 
and soil microbial diversity patterns across an elevation gradient from the Andes to the Amazon. bioRxiv, 079996. doi: $10.1101 / 079996$

Oliveras, I., Bentley, L., Fyllas, N. M., Gvozdevaite, A., Shenkin, A. F., Prepah, T., ..., \& Schwantes Marimon, B. (2020). The influence of taxonomy and environment on leaf trait variation along tropical abiotic gradients. Frontiers in Forests and Global Change, 3, 18. doi: 10.3389 /ffgc. 2020.00018

Peters, M. K., Hemp, A., Appelhans, T., Becker, J. N., Behler, C., Classen, A., ..., \& Gebert, F. (2019). Climate-land-use interactions shape tropical mountain biodiversity and ecosystem functions. Nature, 568(7750), 88-92. doi: 10.1038/s41586-019-1048-z

Pierre, S., Hewson, I., Sparks, J. P., Litton, C. M., Giardina, C., Groffman, P. M., \& Fahey, T. J. (2017). Ammonia oxidizer populations vary with nitrogen cycling across a tropical montane mean annual temperature gradient. Ecology, 98(7), 1896-1907. doi: 10.1002/ecy.1863

R Core Team (2021). R: A language and environment for statistical computing. R Foundation for Statistical Computing, Vienna, Austria. URL https://www.R-project,org/.

Ren, C., Zhang, W., Zhong, Z., Han, X., Yang, G., Feng, Y., \& Ren, G. (2018). Differential responses of soil microbial biomass, diversity, and compositions to altitudinal gradients depend on plant and soil characteristics. Science of The Total Environment, 610-611, 750-758. doi: 10.1016/j.scitotenv.2017.08.110

Rolland, C. (2003). Spatial and Seasonal Variations of Air Temperature Lapse Rates in Alpine Regions. Journal of Climate, 16, 1032-1046. doi: 10.1175/1520-0442(2003)016<1032:SASVOA>2.0.CO;2

Rosado, B. H. P., Joly, C. A., Burgess, S. S. O., Oliveira, R. S., \& Aidar, M. P. M. (2015). Changes in plant functional traits and water use in Atlantic rainforest: evidence of conservative water use in spatio-temporal scales. Trees, 30(1), 47-61. doi: 10.1007/s00468015-1165-8

Sidor, C. G., Popa, I., Vlad, R., \& Cherubini, P. (2015). Different treering responses of Norway spruce to air temperature across an altitudinal gradient in the Eastern Carpathians (Romania). Trees, 29(4), 985-997. doi: 10.1007/s00468-015-1178-3

Soethe, N., Wilcke, W., Homeier, J., Lehmann, J., \& Engels, C. (2008). Plant growth along the altitudinal gradient_ role of plant nutritional status, fine root activity, and soil properties. In Gradients in a tropical mountain ecosystem of Ecuador (pp. 259-266). Springer, Berlin, Heidelberg. doi: 10.1007/978-3-540-73526-7_24

Terra, M. D. C. N. S., Mello, J. M. D., Mello, C. R. D., Santos, R. M. D., Nunes, A. C. R., \& Raimundo, M. R. (2015). Influência topoedafo-climática na vegetação de um fragmento de Mata Atlântica na Serra da Mantiqueira, MG. Revista Ambiente \& Água, 10(4), 928942. doi: 10.4136/ambi-agua.1705

Villar, J. C. E., Ronchail, J., Lavado, W., Carranza, J., Cochonneau, G., De Oliveira, E. U. R. I. D. E. S., \& Pombosa, R. (2010). Variabilidad espacio-tiemporal de las lluvias en la cuenca amazónica y su relación con la variabilidad hidrológica regional. Un enfoque particular sobre la región andina. Revista Peruana GeoAtmosférica, 2, 99-130.

Wang, H. C., Chou, C. Y., Chiou, C. R., Tian, G., \& Chiu, C. Y. (2016). Humic acid composition and characteristics of soil organic matter in relation to the elevation gradient of moso bamboo plantations. PLOS ONE, 11(9). doi: 10.1371/journal.pone.0162193

Wang, Z., Yang, B., Deslauriers, A., \& Bräuning, A. (2014). Intra-annual stem radial increment response of Qilian juniper to temperature and precipitation along an altitudinal gradient in northwestern China. Trees, 29(1), 25-34. doi: 10.1007/s00468-014-1037-7

Whitaker, J., Ostle, N., Nottingham, A. T., Ccahuana, A., Salinas, N., Bardgett, R. D., ..., \& McNamara, N. P. (2014). Microbial community composition explains soil respiration responses to changing carbon inputs along an A ndes-to-A mazon elevation gradient. Journal of Ecology, 102(4), 1058-1071. doi: 10.1111/13652745.12247

Wilcke, W., Oelmann, Y., Schmitt, A., Valarezo, C., Zech, W. y Homeier, J. (2008). Soils along the altitudinal transect and in catchments. In Gradients in a Tropical Mountain Ecosystem of Ecuador (pp. 75-85). Springer, Berlin, Heidelberg. doi: 10.1007/978-3-540-73526-7_9

Zhu, Z. X., Nizamani, M. M., Sahu, S. K., Kunasingam, A., \& Wang, H. F. (2019). Tree abundance, richness, and phylogenetic diversity along an elevation gradient in the tropical forest of Diaoluo Mountain in Hainan, China. Acta Oecologica, 101, 103481. doi: 10.1016/j.actao.2019.103481

\section{Manuscrito recibido el 05de octubre de 2020}

Aceptado el 19 de abril de 2021

Publicado el 30 de diciembre de 2021

\section{Este documento se debe citar como:}

Murga-Orrillo, H., Coronado Jorge, M. F., Abanto-Rodríguez, C., \& Lobo, F. A. (2021). Gradiente altitudinal y su influencia en las características edofoclimáticas de los bosques tropicales. Madera y Bosques, 27(3), e2732271. doi: 10.21829/myb.2021.2732271 Commons Atribución-NoComercialCompartirlgual 4.0 Internacional. 